
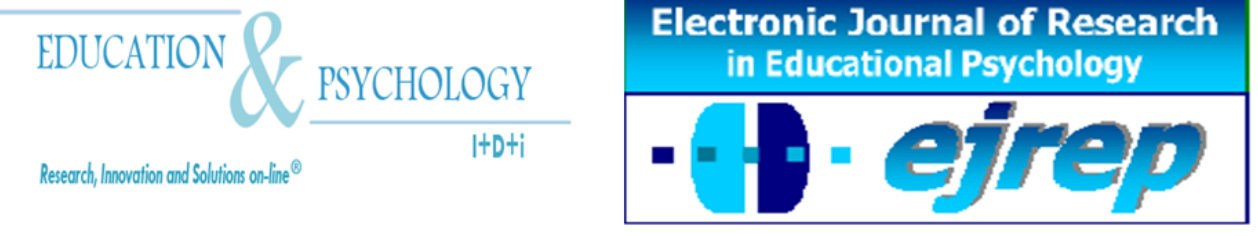

Editorial EOS

\title{
Emotional Intelligence in Physical and Mental Health
}

\section{Agustín Ernesto Martínez González ${ }^{1}$, José Antonio Piqueras ${ }^{2}$, Victoriano Ramos Linares ${ }^{3}$}

\author{
${ }^{1}$ Departamento de Investigación y Tratamiento, \\ Clínica Neuropsicológica Mayor, Alcantarilla (Murcia) \\ ${ }^{2}$ Departamento de Psicología de la Salud, \\ Universidad Miguel Hernández de Elche (Alicante) \\ ${ }^{3}$ Servicios Sociales de la Mancomunitat Marina Baixa (Alicante)
}

\section{Spain}

Correspondence: Agustín Ernesto Martínez González. C/Mayor, 113, 4C. 30820 Alcantarilla (Murcia). Spain. E-mail: cpcmayor@yahoo.es

(C) Education \& Psychology $\mathrm{I}+\mathrm{D}+\mathrm{i}$ and Editorial EOS (Spain) 


\begin{abstract}
During the last decade, interest in the study of Emotional Intelligence and its relationship with health has increased considerably. This article aims to display the results of relevant studies linking Emotional Intelligence, as a trait, and physical and mental health condictions. Results indicate that high levels of emotional intelligence are significantly associated with a good mental health, while low levels of emotional intelligence are related with certain psychological disorders. Similarly, Emotional Intelligence seems to play a moderating role on the symptoms and medical conditions. Future studies should deepen knowledge of the relationship between emotional intelligence and psychological variables (i.e., personality, prosocial behavior, etc.), Emotional Intelligence and medical conditions (i.e., chronic pain, cancer, etc.).
\end{abstract}

Keywords: perceived emotional intelligence, trait emotional intelligence, health, wellness, mental health, physical health.

Received: 01/28/09 Initial Acceptance: 02/01/09_Definitive Acceptance: 04/27/10

\title{
Inteligencia Emocional en la Salud Física y Mental
}

\section{Resumen}

A lo largo de esta última década el interés por el estudio de la Inteligencia Emocional y su relación con la salud ha aumentado notablemente. El presente artículo tiene el objetivo de presentar los resultados de los estudios más relevantes que relacionan la Inteligencia Emocional medida como rasgo con las condiciones de salud física y mental. Los resultados indican que niveles altos de Inteligencia Emocional están significativamente relacionados con una buena salud mental mientras que niveles bajos de Inteligencia Emocional se relacionan con ciertos trastornos psicológicos. Del mismo modo, la Inteligencia Emocional parece jugar un papel moderador sobre los síntomas y enfermedades médicas. Futuros estudios deberían profundizar en el conocimiento de la relación entre Inteligencia Emocional y variables psicológicas implicadas en la salud mental (por ejemplo, dimensiones de personalidad, conducta prosocial, etc.) y entre Inteligencia Emocional y enfermedades médicas (por ejemplo, dolor crónico, cáncer, etc.).

Palabras clave: inteligencia emocional percibida, inteligencia emocional rasgo, salud, bienestar, salud mental, salud física.

Recibido: 28/01/10 Aceptación Inicial: 01/02/10 Aceptación Definitiva: 27/04/10 


\section{Introducción}

Durante el siglo XX el foco de interés de la psicología de la salud ha sido fundamentalmente la enfermedad y los factores de riesgo asociados como el estrés (Ramos, Rivero, Piqueras, García-López y Oblitas, 2008; Rivero, Piqueras, Ramos, García-López, Martínez y Oblitas, 2008) o las emociones negativas (Piqueras, Martínez, Ramos, Rivero, García-López y Oblitas, 2008; Piqueras, Ramos, Martínez y Oblitas, 2009), el patrón de conducta tipo A (Friedman y Rosenman, 1974), el locus de control (Rotter, 1966), la baja autoestima (Rector y Roger, 1997) y los estilos de personalidad (Ramos et al., 2008; Rivero et al., 2008). Sin embargo, recientemente ha surgido un interés cada vez mayor hacia el estudio de la relación entre el proceso de salud-enfermedad y variables "positivas" tales como la inteligencia emocional, la competencia social o la conducta prosocial (Martínez, 2009).

El concepto de inteligencia emocional (IE en adelante) surgió a comienzos de la década de los 90 (Salovey y Mayer, 1990), como un constructo que incluye diferentes aspectos que van desde la empatía hasta la expresión de las emociones, pasando por el autocontrol y la resolución de problemas entre otros. Concretamente, según estos mismos autores la expresión inteligencia emocional se define como "la capacidad para supervisar los sentimientos y las emociones de uno/a mismo/a y de los demás, de discriminar entre ellos y de usar esta información para la orientación de la acción y el pensamiento propios" (Salovey y Mayer, 1990, p.189).

Este término incluye 2 constructos de por sí suficientemente complejos para merecer unas líneas de atención. En primer lugar, la inteligencia puede ser entendida como la habilidad de comprender ideas complejas, de adaptarse eficazmente al entorno, de aprender de la experiencia, de encontrar varias formas de razonar o de superar obstáculos mediante la reflexión (Neisser et al., 1996). En segundo lugar, el concepto de emociones alude a reacciones psicofisiológicas de las personas ante situaciones relevantes desde un punto de vista adaptativo, tales como aquellas que implican peligro, amenaza, daño, pérdida, éxito, novedad, etc. Estas reacciones son de carácter universal, bastante independientes de la cultura, producen cambios en la experiencia afectiva (dimensión cognitivo-subjetiva), en la activación fisiológica (dimensión fisiológica-adaptativa) y en la conducta expresiva (dimensión conductualexpresiva). Además, poseen un sustrato biológico considerable, son esencialmente agradables 
o desagradables, nos activan y forman parte de la comunicación con los demás y pueden actuar como potentes motivos de la conducta (Cano-Vindel y Miguel-Tobal, 2001).

Así, la IE ha sido operacionalizada de dos modos: como una habilidad similar a la inteligencia cognitiva, es decir, como habilidad o ejecución, y como un rasgo. La combinación de ambos conceptos han dado lugar a la expresión IE, que implica una adecuada gestión de las emociones que permite al individuo afrontar adecuadamente los imperativos prácticos de la vida. Es por ello, por lo que la teoría de la IE propuesta por Salovey y Mayer nos facilita un nuevo marco para investigar y comprender los mecanismos de adaptación emocional ante situaciones de estrés.

Mayer, Salovey y Caruso (2000) definieron la IE como una forma de inteligencia social, separada de la inteligencia general y que tiene relación con la emoción y la cognición. El modelo de IE de Mayer, Salovey y Caruso (2004) posee 4 características principales: 1) la habilidad para percibir las emociones propias y de los demás; 2) la habilidad para utilizar las emociones con el fin de facilitar la toma de decisiones; 3) la habilidad para conocer las emociones; y 4) la habilidad para regular las emociones propias y de los demás. Mayer, Salovey y Caruso (2004) conciben la IE como una habilidad similar a la inteligencia cognitiva. De acuerdo con esta conceptualización basada en el modelo de habilidad o ejecución dichos autores desarrollaron la Escala Multifactorial de Inteligencia Emocional (Multifactor Emotional Intelligence Scale, MEIS; Mayer, Caruso y Salovey, 1999) que posteriormente tuvo como sucesora el Test de Inteligencia Emocional Mayer-Salovey-Caruso (Mayer-Salovey-Caruso Emotional Intelligence Test, MSCEIT; Mayer, Salovey, Caruso, y Sitarenios, 2003), adaptada al castellano por Extremera, Fernandez-Berrocal y Salovey (2006). Se han desarrollado dos versiones, el MSCEIT v.1.1. y el MSCEIT v.2.0. Esta última es una versión más corta diseñada para el ámbito profesional e investigador. El MSCEIT v.2.0. consta de ocho tareas diferentes (141 ítems en total), dos para cada uno de los cuatro componentes de la inteligencia emocional. Así, el primer componente, la percepción de las emociones, se evalúa mediante la presentación de fotografías de una persona y pidiendo que se estime el nivel de tristeza, felicidad, miedo, etc., (Percepción emocional en rostros) así como de diversos paisajes y diseños abstractos (Percepción emocional en dibujos). La capacidad de gestión de las emociones (asimilación emocional) se evalúa solicitando del sujeto que indique en qué grado considera que determinados estados de humor como el aburrimiento o la felicidad pueden ser útiles para realizar ciertas actividades. La parte del test dedicada a la comprensión de las emociones in- 
cluye entradas en las que los participantes deben completar frases que evalúan su conocimiento sobre el vocabulario relacionado con las emociones y sobre la evolución y cambio de éstas. El cuarto componente, mide la habilidad para manejar emociones, es decir, el control de las emociones. Esta capacidad es evaluada por una serie de escenarios en las que las personas deben elegir la manera más adaptativa de regular sus propios sentimientos (Auto-manejo emocional) y los sentimientos que surgen en situaciones sociales y con otras personas (Manejo social).

Por otra parte, otros autores han conceptuado la IE como un Rasgo (Neubauer y Freudenthaler, 2005; Petrides y Furnham, 2001) similar a las características de personalidad. En este sentido, el modelo mixto de la IE hace referencia a las habilidades mentales, destrezas o competencias y las características de personalidad. Esta propuesta fue materializada por medio de diversas escalas como el Trait Meta-Mood Scale (TMMS; Salovey, Mayer, Goldman, Turvey y Palfai, 1995) o el Emotional Quotient Inventory (EQ-I; Bar-on, 2000). Esta última, no necesariamente describe las medidas como rasgo, sino más bien como destrezas o competencias. Mientras el TMMS es una de las escalas más utilizadas, existiendo una versión reducida en castellano (TMMS-24; Extremera y Fernández-Berrocal, 2005; Fernández-Berrocal, Extremera y Ramos, 2004). El TMMS es una escala rasgo de metaconocimiento de los estados emocionales que, en su versión extensa, evalúa, a través de 48 ítems, las diferencias individuales en las destrezas con las que los individuos son conscientes de sus propias emociones, así como su capacidad para regularlas. El TMMS-48 mide en una escala de tipo Likert de 5 puntos tres dimensiones claves en la IE: "Atención a los sentimientos", "Claridad emocional" y "Reparación de las emociones". La subescala "Atención a los sentimientos" refleja el grado en el que las personas creen prestar atención a sus emociones y sentimientos. "Claridad emocional" se refiere a cómo las personas creen percibir sus emociones. Por último, "Reparación de las emociones" alude a la creencia del sujeto en su capacidad para interrumpir y regular estados emocionales negativos y prolongar los positivos. Sin embargo, algunos autores indican que esta prueba solamente hace referencia a las percepciones que tienen las personas sobre sus propias habilidades emocionales más que a los niveles reales de IE. Por lo que sería más correcto denominar las puntuaciones del TMMS como índice de Inteligencia Emocional Percibida (IEP) para diferenciarlo de las pruebas de ejecución o habilidad (Salovey, Stroud, Woolery y Epel, 2002). En cuanto a la capacidad predictiva del TMMS-48, ésta ha sido ampliamente demostrada (Extremera y Fernández-Berrocal, 2002; Salovey et al., 2002). 


\section{Objetivo}

En el presente artículo pretendemos realizar una revisión sobre los resultados hallados por los estudios sobre IE medida como rasgo y el proceso salud-enfermedad mental y física, con el fin de mostrar una síntesis del estado actual de la cuestión.

La búsqueda bibliográfica se realizó mediante diversas bases de datos y buscadores SCOPUS. Los términos introducidos para la realización de la búsqueda fueron: emotional intelligence and health y estos términos en español. La búsqueda ofreció 576 resultados en inglés y 25 documentos en español. Tras revisar los resúmenes de cada uno de los artículos, se seleccionaron tan solo los estudios que medían la IE como rasgo, siendo el total de 48 estudios, que fueron solicitados a los autores o descargados de diferentes páginas que ofrecen la posibilidad de realizar descargas de texto completo. Solamente se consideraron los estudios que hallaron un nivel de significación inferior a 0,05 y los estudios que median la IE como rasgo (por ejemplo, TMMS, EQ-I). En el diseño de la revisión seguimos la línea de otros autores que creen que una tabulación descriptiva de los resultados de los estudios individualmente proporciona más perspectiva que un meta-análisis (Kuelz, Hohagen, Voderholzer, 2004).

\section{Desarrollo}

\section{Salud Mental e Inteligencia Emocional}

Numerosos autores han destacado que las habilidades para identificar, comprender y regular las emociones y la de los demás son esenciales para adaptarse a situaciones estresantes vitales (Adeyemo, 2008; Matthews, Zeidner y Roberts, 2002; Salovey, Bedell, Detweiler y Mayer, 1999). La puntuaciones altas en IE del TMMS-48 se han asociado con una mejor recuperación ante eventos negativos (Salovey et al., 1995); menor nivel de estrés ante los exámenes o estresores de tipo experimental (Goldman, Kraemer, y Salovey, 1996; Mikolajczak, Petrides, Coumans y Luminet, 2009; Salovey, Stroud, Woolery, y Epel; 2002); mayor satisfacción vital, menor alexitimia y puntuaciones más bajas en sintomatología rumiativa, ansiosa y depresiva (Fernández-Berrocal, Ramos y Extremera 2001; Fernández-Berrocal, Ramos y Orozco, 1999; Martinez-Pons, 1997); mayor empatía, optimismo y mejor calidad en las relaciones interpersonales (Salovey et al., 2002) y satisfacción sexual (Burri, Cherkas y Spector, 
2009), menor número de pensamientos de venganza y de enfado tras un conflicto (Sukhodolsky, Golub y Cromwell, 2001) y menor uso desadaptativo de Internet y el teléfono móvil (Beranuy, Oberst, Carbonell, y Chamarro (2009).

Un meta-análisis reciente realizado en 7898 sujetos encontró que niveles altos de IE estaban significativamente relacionados con una buena salud mental (Schutte, Malouff, Thorsteinsson, Bhullar, y Rooke, 2007). Por otra parte, algunas investigaciones han constatado que los niveles bajos de IE se relacionan con ciertos desajustes emocionales como la sintomatología depresiva (Fernández- Berrocal, Ramos y Orozco, 1999; Salovey, Stroud, Woolery y Epel, 2002), la alexitimia o dificultad para expresar verbalmente sentimientos y escasa conciencia emocional (Lumley, Gustavson, Partridge y Labouvie-Vief, 2005), niveles mayores de ansiedad (Salovey et al., 2002), trastornos de personalidad (Leible y Snell, 2004), conducta antisocial (Moriarty, Stough, Tidmarsh, Eger y Dennison, 2001), trastornos de la alimentación, como la bulimia (Costarelli, Demerzi y Stamou, 2009; Markey y Vander-Wal, 2007; Pettit, Jacob, Page y Porras, 2010) y sintomatología somática (Augusto-Landa y Montes-Berges, 2009).

\section{a) Ansiedad y Depresión}

A continuación presentamos algunos estudios relacionados con la IE y la sintomatología ansiosa y depresiva. Inicialmente, fue Nolen-Hoeksema $(1991,2000)$ quien propuso una teoría en la cual describió el modo en el que los sujetos manejaban sus sentimientos y pensamientos en momentos de tristeza, este manejo influía en la severidad y duración de los síntomas depresivos y ansiógenos. Así, un estilo de afrontamiento basado en un estilo rumiativo prolongó los síntomas depresivos, mientras que un estilo basado en la realización de actividades distractoras o búsqueda de resolución de problemas aliviaban los síntomas de tristeza (Nolen-Hoeksema, 1991; 2003). Del mismo modo, indicadores de IE estuvieron muy relacionados con la resolución de problemas y el trabajo en equipo (Augusto, Aguilar-Luzón, y Salguero de Ugarte, 2008; Jordan y Troth, 2004; Saklofske, Austin, Galloway y Davidson, 2007).

En efecto, alguno de los primeros estudios como el de Martínez-Pons (1997) comprobaron el poder predictivo de la IE en áreas de funcionamiento personal como: las metas (de dominio y de éxito competitivo); la satisfacción vital (calidad de vida general) y la sintomato- 
logía depresiva. Este autor halló que la IE correlacionó positivamente con la satisfacción vital y las metas de dominio, mientras que lo hizo negativamente con síntomas depresivos. Además, la influencia de la IE sobre la satisfacción vital estuvo mediatizada por la variable de dominio de las tareas y la influencia de la IE sobre la depresión también fue indirecta a través de la percepción de satisfacción vital. En conclusión, cuanto más elevada era la IE mayor era la capacidad para realizar comportamientos adaptativos orientados hacia tareas, mientras que los niveles elevados en estas dos áreas mejoraban la percepción sobre la calidad de vida, disminuyendo la sintomatología depresiva.

En consonancia con estos resultados, a lo largo de los años noventa comenzaron a surgir una serie de estudios que destacaron la relación significativa entre salud mental e IE (Fernández-Berrocal y Ramos-Díaz 1999; Goldman et al., 1996; Martinez-Pons, 1999; Schutte et al., 1998). Así, Fernández-Berrocal y Ramos-Díaz (1999) llegaron a la conclusión de que la IE predecía el ajuste psicológico y que la IE era un concepto básico para entender cómo variables disposicionales se relacionaban con el estrés, el afrontamiento y la adaptación (Saklofske et al., 2007).

Los primeros estudios en población de habla hispana han destacado que tanto la reparación como la claridad correlacionaron negativamente y de forma significativa con el Inventario de Beck para la Depresión (BDI). Las mujeres con un estado anímico normal se diferenciaron de las mujeres depresivas por tener puntuaciones menores en las escalas de atención a los sentimientos, mayores en claridad y reparación de los estados emocionales de la escala TMMS. Por lo tanto, las mujeres con una puntuación alta en las escalas de reparación y claridad del TMMS informaron de niveles más bajos de depresión (Fernández-Berrocal, Ramos y Orozco, 1999).

En el nuevo siglo, ha habido un aumento exponencial del número de estudios transculturales. La mayoría estos estudios internacionales y nacionales han indicado una relación alta y significativa entre IE y salud mental (Austin et al., 2005; Bastian et al., 2005; Borges, Stratton, Wagner y Elam, 2009; Brown y Schutte, 2006; Brackett y Mayer, 2003; Ciarrochi et al., 2001; Ciarrochi et al., 2002; Day et al., 2005; Dawda y Hart, 2000; Donaldson-Feilder y Bond, 2004; Dulewicz et al., 2003; Extremera y Fernández-Berrocal, 2002; Extremera y Fernández-Berrocal, 2005; Fernández-Berrocal et al., 2004; Ghorbani et al., 2002; Hemmati et al., 2004; Jain y Sinha, 2005; Leible y Snell, 2004; Oginska-Bulik, 2005; Pau y Croucher, 
2003; Riley y Schutte, 2003; Salovey et al., 2002; Saklofske et al., 2003; Schmidt y Andrykowski, 2004; Slaski y Cartwright, 2002; Tsaousis y Nikolaou, 2005; Yan et al., 2008).

Así, Gohm, Baumann y Sniezek (2001) investigaron el papel de las experiencias emocionales y las dificultades cognitivas en situaciones de estrés agudo en un grupo de bomberos aplicando el TMMS. Los resultados del estudio revelaron que los bomberos que presentaban mayores niveles de claridad emocional informaban de un menor número de dificultades cognitivas (quedarse en blanco, etc.) durante las simulaciones de fuego. Así, las personas que tenían clara su reacción emocional ante una situación de estrés agudo invertían menos tiempo rumiando en su respuesta emocional, por lo que le permitía prestar más atención a la tarea que estaba realizando. Este mismo autor, encontró que la IEP se asociaba con mayores niveles de felicidad actual, mayor porcentaje de felicidad previa, niveles más elevados de afecto positivo, mayor satisfacción vital y altas puntuaciones en autoestima (Gohm y Clore, 2002a). Asimismo, los niveles en el TMMS se relacionaron de forma inversa con ansiedad general y ansiedad social. Además, las puntuaciones en el TMMS también se asociaron de forma significativa con el estilo atribucional. Así, los estudiantes que tenían una alta puntuación en el TMMS tendían a hacer atribuciones adaptadas de éxito hacia su vida. Por otra parte, las puntuaciones altas en el TMMS se relacionaron de forma significativa con estrategias de reinterpretación positiva y crecimiento, mayor afrontamiento positivo, planificación de la acción y mayor búsqueda de apoyo social y emocional. Así, las personas con alta IEP tendían a centrarse y a desahogar sus emociones pero no a evitar la situación estresante mediante conductas o supresión mental o cognitiva (Gohm y Clore, 2002a).

Por lo tanto, la revisión sobre los estudios hasta la fecha ha indicado que las personas que tenían una alta atención a sus emociones acompañada de niveles elevados de claridad y reparación emocional, poseían un mayor procesamiento emocional de la información. Sin embargo, cuando una alta atención a las emociones no se mantenía en equilibrio con unos adecuados niveles de claridad o reparación, aparecían pensamientos rumiativos y estados emocionales negativos relacionados con la depresión (Extremera y Fernández-Berrocal, 2005; Schutte et al., 2007; Thayer et al., 2003).

En este sentido, parece ser que fue la reparación y no la claridad la que moderó las relaciones existentes entre atención y bienestar emocional. En consecuencia, en los individuos con alta reparación emocional, una elevada atención emocional era beneficiosa para bienestar 
emocional, mientras que los que tenían una baja reparación emocional y una elevada atención les afectaba negativamente en su bienestar emocional (Lischetzche y Eid, 2003). Así, parece ser que las personas deprimidas se caracterizaron por presentar pensamientos rumiativos por lo que puntuaron más en la supresión de pensamientos y obtuvieron menos puntuaciones en atención y claridad emocional (Rude y McCarthy, 2003).

Estudios posteriores han hallado una relación entre la baja ansiedad, la claridad y la reparación emocional y la alta percepción de la salud y el bienestar (Latorre y Montañez, 2004). En esta misma línea, Williams, Fernández-Berrocal, Extremera, Ramos y Joiner (2004) estudiaron la relación entre el factor de reparación del TMMS con los síntomas de depresión endógena y depresión por desesperanza medida mediante el BDI. Estos autores observaron que las relaciones entre regulación emocional y depresión por desesperanza eran más intensas que entre regulación emocional y depresión endógena. Igualmente, la habilidad para regular las emociones y la depresión endógena fue menos intensa en comparación con la depresión por desesperanza. Del mismo modo, otros estudios han hallado una relación positiva entre claridad y apoyo social y salud mental, siendo la claridad y la reparación emocional predictoras de apoyo social y salud mental (Montes-Berges y Augusto, 2007).

Recientemente otros estudios han llegado a resultados similares. La atención emocional se relacionó positivamente con la sintomatología ansiosa y depresiva y negativamente con el rol emocional, funcionamiento social y salud mental. En cambio, la claridad y reparación emocional se relacionaron negativamente con ansiedad y depresión, y positivamente con el físico, funcionamiento social, salud mental, vitalidad y percepción de salud. De igual modo, se ha llegado a confirmar el carácter predictivo de la atención, claridad y reparación emocional sobre los niveles de ansiedad y depresión y áreas relacionadas con la salud mental, social y física (Extremera y Fernández-Berrocal, 2006).

Fernández-Berrocal, Alcalde, Extremera y Pizarro (2006) analizaron la relación entre la IEP y la ansiedad, depresión, medidas de supresión de pensamientos y autoestima en adolescentes. Los resultados indicaron que la reparación emocional se relacionó positivamente con la autoestima y que la IEP correlacionó negativamente con la depresión y la ansiedad. Mientras tanto, la claridad y reparación emocional estuvieron asociadas a un mejor ajuste psicológico, independiente de los efectos de la autoestima y la supresión de pensamientos. 
En otro estudio llevado acabo con adolescentes encontraron que los adolescentes "normales" se diferenciaban de los “disfóricos" por su menor puntuación en atención emocional, ansiedad y supresión de pensamientos. Al mismo tiempo tenían unos índices de afrontamiento, regulación del estado de ánimo y utilización del apoyo social más elevados (Velasco, Fernández, Páez y Campos, 2006).

A pesar de estos datos, todavía son escasos los trabajos que han mostrado que las personas emocionalmente inteligentes utilicen estrategias de afrontamiento más efectivas para manejar sus sentimientos negativos (Matthews, Zeidner y Roberts, 2002).

Por otra parte, del grupo de estudios que han analizado las relaciones entre IE y ansiedad y depresión, algunos autores han remarcado la importancia de las diferencias culturales y/o de género como variables mediadoras de esta relación. Así, algunos estudios transculturales han encontrado una asociación entre depresión e inteligencia emocional percibida, género y dimensiones culturales (Extremera y Fernández-Berrocal, 2002; Fernández-Berrocal, Ramos y Extremera, 2001; Salovey et al., 2000; 1995; 2002). Parece ser que los países con culturas más individualistas presentaron una expresión y experiencia de emociones negativas más intensas (Fernández, Carrera, Sánchez, Paez, y Candía, 2000; Fernández-Berrocal et al., 2005).

En cuanto a las diferencias de género y edad, la mayoría de los estudios han indicado que las mujeres puntuaban ligeramente más altos que los hombres en IE, y que las puntuaciones tendían a incrementar con la edad (Thayer, Rossy, Ruiz-Padial y Johnsen, 2003; VanRooy, Alonso y Viswesvaran, 2005). Sin embargo, algunos estudios han encontrado que las mujeres presentaban mayores síntomas depresivos y mayores niveles de atención a sus emociones, al igual que menos capacidad de reparación emocional. No obstante, estos datos no son concluyentes y son necesarios más estudios (Fernández-Berrocal, Ramos y Extremera, 2001; Lynn y Martin, 1997; Nolen-Hoeksema, Larson y Grayson, 1999).

En resumen, la IE parece actuar como un protector en la salud mental e influir en la disminución de la vulnerabilidad hacia los estados emocionales negativos y la depresión. Las investigaciones utilizando el TMMS muestran que las dimensiones de claridad y reparación se relacionan negativamente con el afecto negativo y la depresión, mientras que la dimensión de 
atención lo hace en sentido positivo en muchos casos (Extremera y Fernández-Berrocal, 2006; Fernández-Berrocal et al., 2006; Rude y McCarthy, 2003).

\section{b) Personalidad}

En el desarrollo del este apartado se han abordado por un lado los estudios centrados en la investigación de los rasgos de la personalidad y la IE y, por otro lado, las investigaciones que estudiaban la relación entre IE y trastornos de personalidad.

Respecto a los estudios que se centran en los rasgos de la personalidad y la IE, la literatura indicaba que las personas con ciertos rasgos de personalidad tenían una mayor tendencia a realizar estrategias de regulación emocional ante situaciones de estrés o depresión (Greven, Chamorro-Premuzic, Arteche y Furnham, 2008; Lam, Smith, Checkley, Rijsdijk y Shan, 2003). Diversos autores han considerado que los rasgos de personalidad de neuroticismo y extraversión influyen en las estrategias de regulación emocional tanto en adultos (Kokkonen y Pulkkinen, 2001) como en adolescentes (Gomez, Holmberg, Bounds, Fullarton y Gomez, 1999). Además, indican que éstos actúan como predictores del procesamiento emocional negativo y positivo (Gomez, Gomez y Cooper, 2002), estando estas características de personalidad muy relacionadas con el uso de estrategias de rumiación o de distracción (Bagby y Parker, 2001; Lam et al., 2003).

Pese al creciente número de investigaciones algunos estudios han encontrado que la evaluación de la IE tiene ciertas similitudes con otros constructos como los rasgos de personalidad. Así, Austin, Saklofske y Egan, (2005) hallaron que la IE estaba fuertemente relacionada con la cantidad de apoyo social, y que a su vez esta claridad de apoyo social y satisfacción con la vida, estaban muy relacionadas con la personalidad. En este sentido, algunos autores consideran que muchas escalas de IE (en el AES: Brackett et al., 2006; MacCann, 2006, en el EQ-I: Austin et al., 2005; Brackett et al., 2006; Dawda y Hart, 2000) correlacionan con las dimensiones de neuroticismo y extraversión de la personalidad. Gannon y Ranzijn (2005) argumentaron que las escalas de IE lo que realmente medían era personalidad. En su investigación llegaron a la conclusión de que existía alguna coincidencia entre personalidad e IE, pero que la IE podía ofrecer interpretaciones valiosas a las teorías actuales de la personalidad. Así pues, algunos autores han defendido que la IE estaría considerada como un rasgo de personalidad (Petrides, Furnham y Frederickson, 2004). 
Respecto a los trastornos de la personalidad, también se han realizado distintas investigaciones. Kerns (2005) estudió la relación entre la esquizotipia positiva y el procesamiento emocional rasgo evaluado con el TMMS y la ejecución de tareas cognitivas. Este autor encontró que el procesamiento emocional rasgo estaba asociado a la esquizotipia positiva, ya que prestaban mayor atención a las emociones, pero tenían menos claridad emocional que el grupo de control. Gohm (2003) encontró que el 63\% de los esquizotípicos tenía una alta intensidad y atención emocional, una tendencia a abrumarse emocionalmente así como una baja claridad emocional.

Por su parte, Leible y Snell (2004) estudiaron la relación entre los factores del TMMS y pacientes con trastorno de la personalidad límite. Estos autores encontraron menores niveles de claridad y de reparación en los pacientes límites por lo que presentaban problemas de adaptación socioemocional. Respecto a otros tipos de trastornos de personalidad, encontraron que la atención emocional correlacionaba negativamente con los antisociales y los esquizoides, mientras que fue positiva con los síntomas histriónicos. La claridad emocional reveló correlaciones negativas con todos los trastornos de personalidad, al igual que el factor de reparación emocional cuya única excepción se encontró en el trastorno de personalidad histriónico.

Por lo tanto, las personas que presentan diagnóstico de trastorno de personalidad se caracterizan por tener una capacidad baja para auto-regularse emocionalmente y afrontar las situaciones de estrés. Esto es así, posiblemente, debido a la dificultad o falta de comprensión de las emociones.

\section{c) Consumo de drogas}

El consumo de drogas es un problema de salud pública mundial. En la última década los estudios sobre IE y consumo de droga han ido en aumento, aunque todavía son escasos. Sin embargo, diversos autores han hallado una relación positiva entre IE y la evitación de consumo de sustancias adictivas. Por lo tanto, la IE parece desempeñar un papel decisivo en la abstinencia de consumo de drogas. Una explicación habitual en el campo científico ha sido que las personas con falta de habilidades emocionales podían recurrir al consumo de sustancias como una forma de autorregular o disminuir sus estados emocionales negativos o como búsqueda de placer o refuerzo que podría generar emociones agradables. 
Los primeros estudios con adolescentes hallaron que los más inteligentes emocionalmente tenían un consumo menor de tabaco y alcohol (Trinidad y Johnson, 2002). Estos resultados revelaron que una baja IE era un factor de riesgo para el consumo de sustancias en la adolescencia. Parece ser que, entre otras explicaciones posibles, los adolescentes emocionalmente inteligentes comprendían y soportaban mejor la presión del grupo de compañeros que les incitaban a consumir y resolvían mejor la discrepancia entre sus motivaciones y las del grupo.

Por lo tanto, parece ser que la IE es un buen protector para evitar la aparición de adicciones. En este sentido, Trinidad, Unger, Chou, Azen y Johnson, (2004a) evaluaron el carácter protector de la IE sobre el riesgo de fumar en los adolescentes. Estos autores hallaron que la IE era un factor de protección de riesgo de fumar ya que se relacionaba con una mejor percepción de las consecuencias sociales negativas asociadas con fumar y con una mayor eficacia para rechazar el ofrecimiento de cigarrillos por parte de compañeros. Los adolescentes con una alta IE manifestaban menos intenciones de fumar el año próximo. Sin embargo, tenían más probabilidades de fumar si ya lo habían intentado con anterioridad. En cambio, los que tenían una baja IE tenían más probabilidad de fumar en el futuro.

Los estudios en población universitaria llegaron a la misma conclusión (Brackett, Mayer y Warner, 2004). El consumo de drogas ilegales y el consumo de alcohol correlacionaron negativamente con la IE. Aquellos individuos que tenían claridad y una capacidad de reparar las emociones tenían una percepción positiva de la salud, incrementando las conductas de salud (actividad física, etc.) (Li, Lu y Wang, 2009) y presentaban menos consumo de alcohol y drogas (Latorre y Montañés, 2004; Queirós, Cancela y Fernández-Berrocal, 2004)

Sin embargo, los sujetos que presentaban niveles bajos en el componente de reparación del TMMS comenzaban a consumir tabaco o cannabis a una edad más temprana (Limonero, Tomas-Sabado y Fernández-Castro, 2006). Así pues, la claridad emocional parece estar relacionada con el consumo ocasional de cannabis, de modo que los estudiantes con alta puntuación en este factor consumen menos. Mientras tanto, el componente de atención emocional del TMMS no parece estar implicado en el consumo de estas sustancias.

\section{Inteligencia emocional y Salud Física}


En cuanto la relación entre IE y salud física, son escasos y muy recientes los estudios que destacan el papel de la IE como protector en el afrontamiento de los síntomas característicos de patologías médicas como el sida, el cáncer, la fibriomialgia y el dolor crónico.

Estudios meta-analíticos (Schutte et al., 2007) y un número considerable de estudios sobre IE han hallado correlaciones significativas entre IE y salud física (Donaldson-Feilder y Bond, 2004; Extremera y Fernandez-Berrocal, 2002; Goldman et al., 1996; Tsaousis y Nikolaou, 2005).

Los primeros estudios que investigaron la relación entre la IE y la salud fueron los de Goldman, Kraemer y Solovey (1996). Estos autores destacaron el papel moderador de la IE sobre los síntomas y enfermedades médicas. En su investigación utilizaron población universitaria y analizaron las puntuaciones del TMMS junto a medidas de estrés, síntomas fisiológicos y visitas al centro de salud durante periodos de estrés general (hacer exámenes). Llegaron a la conclusión que la adaptación al estrés dependía de la capacidad para atender, discriminar y regular sentimientos. Así, si el estrés era considerable, los alumnos con mayor atención a los sentimientos tenían más probabilidad de informar de síntomas físicos, mientras que los que regulaban sus emociones presentaban, en momentos de estrés, menos informes de enfermedad y de visitas al médico.

Schmidt y Andrykowski (2004) estudiaron la influencia de la IEP, el apoyo social y las restricciones sociales en el ajuste psicológico de 210 mujeres con cáncer de mama. Las mujeres con altas restricciones sociales, que ocultaban sus emociones con respecto al cáncer, presentaban una baja IEP por lo que sufrían más malestar, más ansiedad y depresión. Así, puede observarse que la IE funcionó como un protector contra el impacto de un ambiente social negativo, desempeñando un papel importante en el proceso de adaptación al cáncer de mama.

Son pocos los estudios que han analizado la relación entre el dolor crónico y la IEP. Zautra, Smith, Affleck y Tennen (2001) estudiaron la relación entre claridad, afecto negativo del TMMS y dolor en enfermos crónicos de artritis y fibriomialgia. Los resultados resaltaron que el afecto positivo redujo la relación entre el afecto negativo y el dolor. Los pacientes con artritis y que tenían una mayor claridad emocional confundieron menos sus estados afectivos 
positivos y negativos. Mientras que los pacientes con fibriomialgia con una alta claridad tuvieron más afecto positivo y menos negativo.

Algunos autores han investigado las relaciones entre IE y el ajuste psicológico de los pacientes que van a ser sometidos a procesos médico-quirúrgicos (Fernández-Berrocal, Ramos y Orozco, 2000). Carranque et al., (2004) hallaron que los pacientes que presentaron niveles más altos de IE (mayor capacidad de comprensión y reparación emocional) evaluaron su dolor postoperatorio como menos intenso. Los análisis de regresión indicaron un efecto significativo en la comprensión y reparación emocional sobre la percepción del dolor postoperatorio. Así pues, varios estudios ponían de manifiesto la presencia de menos dolor postoperatorio en aquellos pacientes con mayor capacidad para la comprensión y reparación de sus emociones (Extremera y Fernández-Berrocal, 2002; Fernández-Berrocal, Ramos y Orozco, 2000; Salovey, 2001) siendo estas capacidades decisivas para el ajuste al estrés provocado por el acto quirúrgico.

\section{Conclusiones}

En este artículo de revisión se han intentado sintetizar los resultados de distintas investigaciones en relación con la IE, medida como rasgo, y la salud mental y física. Una de las conclusiones obtenida es que la IEP medida mediante autoinforme parece estar relacionada con la salud y el bienestar de las personas.

Respecto a los estudios que han relacionado IE y salud mental, podemos indicar que existe un número creciente de investigaciones que concluyen que niveles altos de IE estan relacionados significativamente con el bienestar emocional y la salud mental. Coherente con este hallazgo es el que indica que niveles bajos de IE se relacionan con ciertos problemas emocionales, tales como depresión, dificultad para verbalizar sentimientos, escasa conciencia emocional, ansiedad, problemas en la personalidad y conductas adictivas. Concretamente en la escala TMMS, las dimensiones de claridad y reparación correlacionan negativamente con la ansiedad y la depresión, mientras que la dimensión atención lo hace en sentido positivo. Así pues, la IE ha sido considerada por diferentes autores como un buen protector frente a la aparición de trastornos psicológicos como la ansiedad o la depresión, ya que disminuye la vulnerabilidad al estrés y evita la aparición de estos trastornos. 
Por otra parte, en relación a los estudios de IE y problemas de salud física o médica, y pese a los escasos estudios realizados, la mayoría de los autores destacan el papel moderador de la IE sobre el afrontamiento de los síntomas de enfermedades médicas. Así, niveles altos de IE o de capacidad de comprensión y reparación emocional se han relacionado con una menor intensidad del dolor postoperatorio. Esto indicaría que la IE podría funcionar como un protector contra acontecimientos estresantes, negativos o aversivos (como sufrir una enfermedad o un proceso operatorio), jugando un papel crucial en el proceso de adaptación del individuo ante la enfermedad.

A tenor de los resultados de los diferentes estudios, estos datos sugieren la importancia de fomentar el desarrollo de la IE desde la niñez para prevenir la aparición de trastornos emocionales y mejorar las estrategias de afrontamiento ante procesos de enfermedad. Coincidimos con la opinión de diversos autores (v.gr., Eack et al., 2007; Moriarty et al., 2001; Nelis et al., 2009; Karahan y Yalcin, 2009; Yalcin, Karahan, Ozcelik y Igde, 2008; Slaski y Cartwright, 2000b; Trinidad et al., 2004a; b) que destacan la necesidad de enseñar y entrenar ciertas habilidades emocionales de la IE. De esta afirmación se deriva la necesidad de trabajar en el desarrollo de intervenciones preventivas y de promoción de la salud desde el enfoque de la inteligencia emociona en el ámbito escolar y familiar. Algunos de los objetivos de estos programas podrían ser los siguientes: (1) dotar de estrategias educativas a los padres y madres mediante seminarios o talleres prácticos (coordinados desde las Asociaciones de Padres y Madres de Alumnos) y (2) fomentar el autocontrol emocional, las habilidades emocionales y sociales desde edades muy tempranas a través de actividades de aprendizaje cooperativo (Martínez, Inglés, Piqueras y Oblitas, en prensa; Martínez, Inglés, Piqueras y Ramos, 2010).

Desde nuestro punto de vista, una aportación de suma importancia sería concretar el entendimiento de la emoción como un concepto compuesto de un estado fisiológico asociado a una cognición; esta perspectiva puede aportar claridad en la compresión de la noción de IE al facilitar la clasificación de las emociones (agradables-alegría/placer-/desagradables -ira, miedo, tristeza-) y por consiguiente facilitar a su vez la toma de decisiones (resolución de problemas) (Ramos, Piqueras, Martínez y Oblitas, 2009).

En definitiva, aunque el concepto IE ha aumentado su popularidad de forma considerable, el campo de estudio de la IE no ha hecho más que dar sus primeros pasos. Consideramos necesario que exista un soporte empírico que demuestre su independencia respecto a 
otros constructos como la personalidad (Johnson, Batey y Holdsworth, 2009), la autoregulación emocional (Lópes et al., 2005) y la conducta prosocial (Martínez, 2009). Por otra parte, coincidimos con otros autores al señalar que la investigación sobre la IE debería desarrollar instrumentos que la evalúen de forma más certera este constructo (Extremera- Pacheco et al. 2004; Pecjak, 2003). Sin embargo, pese a estas limitaciones no es menos cierto que la evidencia empírica con la que cuenta la comunidad científica refleja el papel significativo que juega la IE en el proceso de salud-enfermedad tanto física como mental. En consecuencia, parece lógico continuar profundizando en el conocimiento de este constructo y otros relacionados (conducta prosocial, personalidad, etc.) por su gran interés desde el punto de vista clínico y científico.

\section{Referencias}

Adeyemo, D.A. (2008). El efecto amortiguador de la inteligencia emocional sobre la adaptación de estudiantes de educación secundaria en transición. Electronic Journal of Research in Educational Psychology,6-3 (2), 79-90.

Augusto, J.M., Aguilar-Luzón, M.C. y Salguero de Ugarte, M.F. (2008). El papel de la IEP y del optimismo-pesimismo disposicional en la resolución de problemas sociales: Un estudio con alumnos de trabajo social. Electronic Journal of Research in Educational Psychology, 15, 6, 363-382.

Augusto-Landa, J.M. y Montes-Berges, B.(2009). Perceived emotional intelligence, health and somatic symptomatology in nursing students. Individual Differences Research, 7(3), 197-211.

Austin, E.J. y Saklofske, D.H. (2005) 'Far too many intelligences? On the communalities and differences between social, practical, and emotional intelligences', en R. Schulze and R.D. Roberts (eds), Emotional Intelligence:An International Handbook (pp. 107-28). Cambridge, MA: Hogrefe \& Huber.

Austin, E.J., Saklofske, D.H. y Egan, V. (2005). Personality, well-being and health correlatos of trait emotional intelligence. Personality and Individual Differences, 38 (3), 547558.

Bagby, R.M. y Parker, J.D.A. (2001).Relation of rumination and distraction with and extraversion in a simple of patients with major depression. Cognitive Therapy and Research, 25, 91-102. 
Bagby, R.M., Parker, J.D. y Taylor, G.J. (1994). The twenty-item Toronto Alexithymia ScaleI.Item selection and cross-validation of the factor structure. Journal of Psychosomatic Research,38, 23-32.

Bar-On, R. (1997). Bar-On Emotional Quotient Inventory: A measure of emotional intelligence. Technical Manual: Multi Health Systems.

Bar-On, R. (2000). Emotional and social intelligence: Insights from the Emotional Quotient Inventory. En R. Bar-On y J.D.A. Parker (Eds.), The handbook of emotional intelligence (pp. 363-388). San Francisco: Jossey-Bass.

Bar-on, R., Brown, J.M., Kikcaldy, B.D. y Thome, E.P (2000). Emotional expression and implications for occupational stress: an application of the Emotional Quotient Inventory (EQ-I). Personality and Individual Differences, 28, 1107-1118.

Bastian, V. A., Burns, N. R. y Nettelbeck, T. (2005). Emotional intelligence predicts life skills, but not as well as personality and cognitive abilities. Personality and Individual Differences, 39, 1135-1145.

Beranuy, M., Oberst, U., Carbonell, X.y Chamarro, A. (2009).Problematic Internet and mobile phone use and clinical symptoms in college students: The role of emotional intelligence. Computers in Human Behavior, 25 (5), 1182-1187.

Borges, N.J., Stratton, T.D., Wagner, P.J. y Elam, C.L. (2009). Emotional intelligence and medical specialty choice: findings from three empirical studies. Medical Education. $43,6,565-572$.

Brackett, M. A. y Mayer, J.D. (2003). Convergent, discriminant, and incremental validity of competing measures of emotional intelligence. Personality and Social Psychology Bulletin, 29, 1147-1158.

Brackett, M.A., Mayer, J.D. y Warner, R.M. (2004). Emotional intelligence and its relation to everyday behaviour. Personality and Individual Differences, 36, 1387-1402.

Brackett, M.A., Rivers, S.E., Shiffman, S., Lerner, N. y Salovey, P. (2006) 'Relating emotional abilities to social functioning: A comparison of self-report and performance measures of emotional intelligence', Journal of Personality and Social Psychology, 91(4): 780-95.

Brown, R. F. y Schutte, N.S. (2006). Direct and indirect relationships between emotional intelligence and subjective fatigue in university students. Journal of Psychosomatic Research, 60, 585-93.

Burri A.V., Cherkas L.M. y Spector T.D. (2009). Emotional intelligence and its association with orgasmic frequency in women. Journal of Sexual Medicine, 6 (7), 1930-1937. 
Cano-Vindel, A. y Miguel-Tobal, J. J. (2001). Emociones y Salud. Ansiedad y Estrés, 7, 111121.

Carranque, G.A., Fernández Berrocal, E., Baena, R., Bazán, B., Cárdenas, R., Herraiz, B. y Velasco. (2004). Dolor postoperatorio e inteligencia emocional Revista Española de Anestesiología y Reanimación. 51, 75-79

Ciarrochi, J., Forgas, J.P. y Mayer, J.D. (Eds.) (2006). Emotional intelligence in everyday life (2 $2^{\mathrm{a}}$ ed.). New York, NY: Psychology Press.

Costarelli, V., Demerzi, M. y Stamou, D. (2009). Disordered eating attitudes in relation to body image and emotional intelligence in young women. Journal of Human Nutrition and Dietetics, 22 (3), 239-245.

Davies, M., Stankov, L. y Roberts, R.D. (1998). Emotional Intelligence in search of an elusive construct, Journal of Personality and Social Psychology, 75, 989-1015.

Dawda, D. y Hart, S. D. (2000). Assessing emotional intelligence: Reliability and validity of the Bar-On emotional quotient inventory (EQ-i) in university students. Personality and Individual Differences, 28, 797-812.

Day, A. L., Therrien, D. L. y Carroll, S. A. (2005). Predicting psychological health: Assessing the incremental validity of emotional intelligence beyond personality, Type A behaviour, and daily hassles. European Journal of Personality, 19, 519-536.

Donaldson-Feilder, E. J. y Bond, F. W. (2004). The relative importance of psychological acceptance and emotional intelligence to workplace well-being. British Journal of Guidance \& Counselling, 32, 187-203.

Dulewicz, V., Higgs, M. y Slaski, M. (2003). Measuring emotional intelligence: Content, construct and criterion-related validity. Journal of Managerial Psychology, 18, 405420.

Duran, A., Extremera, N. y Rey, L. (2004). Self-reported emotional intelligence, burnout and engagement among staff in services for people with intellectual disabilities. Psychological Reports, 95, 386-390.

Eack, S.M., Hogarty, G.E., Greenwald, D.P., Hogarty, S.S. y Keshavan, M.S. (2007). Cognitive enhancement therapy improves emotional intelligence in early course schizophrenia: Preliminary effects. Schizophrenia Research, 89, 308-311.

Extremera, N y Fernández- Berrocal, P. (2002). Relation of perceived emotional intelligence and health-related quality of life in middle-aged women. Psychological Report, 91, 47-59. 
Extremera, N. y Fernández-Berrocal, P. (2005). Inteligencia emocional percibida y diferencias individuales en el metaconocimiento de los estados emocionales: una revisión de los estudios con el Trait Meta-Mood Scale. Ansiedad y estrés, 11, 101-122.

Extremera, N. y Fernández-Berrocal, P. (2006). Emotional intelligence as predictor of mental, social, and psysical health in university students. The Spanish Journal of Psychology, 9, 45-51.

Extremera, N., Fernández- Berrocal, P. y Duran, A. (2003).Inteligencia emocional y burnout en profesores. Encuentros en psicología social, 1, 260-265.

Extremera, N., Fernández-Berrocal, P. y Salovey, P. (2006). Spanish version of the MayerSalovey-Caruso Emotional Intelligence Test (MSCEIT) Version 2.0: Reliabilities, Age, and Gender Differences. Psicothema, 18, 42-48.

Extremera, N., Fernández-Berrocal, P., Mestre Navas, J.M, y Guil Bozal, R. (2004). Medidas de evaluación de la inteligencia emocional. Revista Latinoamericana de Psicología, 36, 209-228.

Fernández, I, Carrera, P., Sánchez, F., Páez, D. y Candia, L. (2000). Differences between cultures in emotional verbal and nonverbal reactions. Psicothema, 12, 83-92.

Fernandez-Berrocal, P., Alcalde, R., Extremera, N. y Pizarro, D.A. (2006).The role of emotional intelligence in anxiety and depression among adolescents. Individual Differences Research, 4, 16-27.

Fernández-Berrocal, P., Extremera, N. y Ramos, N.S. (2004). Validity and reliability of the spanish modified version of the Trait Meta-Mood Scale. Psychological Reports, 94, 751-755.

Fernández-Berrocal, P., Ramos, N. y Extremera, N. (2001) Inteligencia emocional, supresión crónica de pensamientos y ajuste psicológico. Boletín de Psicología, 70, 79-95.

Fernández-Berrocal, P., Ramos, N. y Orozco, F. (2000). Inteligencia emocional y estado depresivo durante el embarazo. Toko-Ginecología Práctica, 59, 1-5.

Fernández-Berrocal, P., Ramos, N.S. y Orozco, F. (1999). La influencia de la inteligencia emocional en la sintomatología depresiva durante el embarazo. To-co Ginecología Práctica, 59, 1-5.

Fernández-Berrocal, P., Salovey, P., Vera, A., Extremera, N. y Ramos, N. (2005). Cultural influences on the relation between perceived emotional intelligence and depression. Revue Internationale de Psychologie Sociale, 18, 91-107.

Fernández-Berrocal, P., y Ramos-Díaz, N. (1999).Investigaciones empíricas en el ámbito de la inteligencia emocional. Ansiedad y Estrés, 5, 247-260. 
Friedman, M. y Rosenman, R. H. (1974). Type A Behaviour and Your Heart. Knopf, New York.

Gannon, N., y Ranzijn, R. (2005). Does emotional intelligence predict unique variante in life satisfaction beyond IQ and personality? Personality and Individual Differences, 38 (6), 1353-1364.

Gerits, L., Derksen, J., y Verbruggen, A.B. (2004). Emotional Intelligence and Adaptive Success of Nurses Caring for People with Mental Retardation and Severe Behavior Problems. Mental Retardation, 42, 2,106-121.

Ghorbani, N., Bing, M. N., Watson, P. J., Davison, H. K. y Mack, D. A. (2002). Self-reported emotional intelligence: Construct similarity and functional dissimilarity of higher order processing in Iran and the United States. International Journal of Psychology, 37, 297-308.

Gohm, C.L. (2003). Mood regulation and emotional intelligence: individual differences. Journal of Personality and Social Psychology, 84, 594-607.

Gohm, C.L. y Clore, G.L. (2002a).Affect as information: An individual-differences approach. En L.Feldman Barrett y P.Salovey (Eds.), The wisdom of feelings: Psychological processes in emotional intelligence (pp.341-359). New York: Guilford.

Gohm, C.L., Baumann, M.R. y Sniezek, J.A. (2001). Personality in Extreme Situations: Thinking (or Not) under Acute Stress. Journal of Research in Personality, 35, 388399.

Goldman, S. L., Kramer, D. T. y Salovey, P. (1996). Beliefs about mood moderate the relationship of stress to illness and symptom reporting. Journal of Psychosomatic Research, 41, 115-128.

Goldman, S.L., Kraemer, D.T. y Solovey, P. (1996).Beliefs about mood moderate the relationship of stress to illness and symptom reporting. Journal of Psychosomatic Research, 41, 155-128.

Goleman, D. (1995). Emotional Intelligence. Bloomsbury, London.

Goleman, D. (1997). Beyond IQ: Developing the Leadership Competencies of Emotional Intelligence. Paper presented at the $2^{\text {nd }}$ International Competency Conference; London, Oct. 1997

Goleman, D. (1998). Working with Emotional Intelligence, New York: Bantam Books.

Gomez, R., Gomez, A. y Cooper, A. (2002). Neuroticism and extraversion as predictors of negative and positive emotional information processing: comparing Eysenck's, Gray's, and Newman's theories. European Journal of Personality, 16, 333-350. 
Gomez, R.L., Holmberg, K., Bounds, J., Fullarton, C. y Gomez, A. (1999). Neuroticism and extraversion as predictors of coping styles during early adolescence. Personality and Individual Differences, 27, 3-17.

Greven, C., Chamorro-Premuzic, T., Arteche, A. y Furnham, A. (2008). A hierarchical integration of dispositional determinants of general health in students: The Big Five, trait Emotional Intelligence and Humour Styles. Personality and Individual Differences, 44 (7), 1562-1573.

Havilland, M.G., Warren, W.L. y Riggs, M.L. (2000).An observer scale to measure alexithymia. Psychosomatics, 41, 385-392.

Jain, A. K. y Sinha, A. K. (2005). General health in organizations: Relative relevance of emotional intelligence, trust, and organizational support. International Journal of Stress Management, 12, 257-273.

Johnson, S.J. Batey, M. y Holdsworth L. (2009). Personality and health: The mediating role of Trait Emotional Intelligence and Work Locus of Control Personality and Individual Differences, 47 (5), 470-475.

Jordan, P.J., y Troth, A.C. (2004). Managing Emotions during Team Problem Solving: Emotional Intelligence and Conflict Resolution. Human Performance, 17(2), 195-218.

Karahan, T.F. y Yalcin, B.M. (2009).The effects of an emotional intelligence skills training program on anxiety, burnout and glycemic control in type 2 diabetes mellitus patients. Turkiye Klinikleri Journal of Medical Sciences, 29 (1), 16-24.

Kerns, J.G. (2005). Positive schizotypy and emotion processing. Journal of Abnormal Psychology, 114, 392-401.

Kokkonen, M. y Pulkkinen, L. (2001).Extraversion and neuroticism as antecedents regulation and dysregulation in adulthood. European Journal of Personality, 15, 407-424.

Kuelz, A.K., Hohagen, F., y Voderholzer, U. (2004). Neuropsychological performance in obsessive-compulsive disorder: a critical review. Biological Psychology, 65, 185-236.

Lam, D., Smith, N., Checkley, S., Rijsdijk, F.y Sham, P. (2003). Effect of neuroticism response style and information processing on depression severity in a clinically sample. Psychological Medicine, 33, 469-479.

Latorre, J.M. y Montañez, J. (2004). Ansiedad, inteligencia emocional y salud en la adolescencia. Ansiedad y Estrés, 10, 29-41.

Leible, T.L. y Snell, Jr., W.E. (2004). Borderline personality disorder and multiple aspects of emotional intelligence. Personality and Individual Differences, 37, 393-404. 
Li, G.S.-F., Lu, F.J.H. y Wang A.H.-H. (2009). Exploring the relationships of physical activity, emotional intelligence and health in Taiwan college students. Journal of Exercise Science and Fitness, 7 (1), 55-63.

Limonero, J.T., Tomas-Sábado, J. y Fernández-Castro J. (2006). Perceived emotional intelligence and its relation to tobacco and cannabis use among university students. Psicothema, 18, 95-100.

Limonero, J.T., Tomas-Sábado, J., Fernández-Castro, J. y Gómez-Benito, J. (2004). Influencia de la inteligencia emocional percibida en estrés laboral de enfermería. Ansiedad y Estrés, 10, 29-41.

Lischetzche, T. y Eid, M. (2003).Is attention to feelings beneficial or detrimental to affective well-being? Mood regulation as a moderator variable. Emotion, 3, 361-377.

Lopes, P. N., Salovey, P., Côté, S. y Beers, M. (2005). Emotion regulation ability and the quality of social interaction. Emotion, 1,113-18.

Lumley, M.A., Gustavson, B.J., Partridge, R.T. y Labouvie-Vief, G. (2005).Assessing alexithymia and related emotional ability constructs using multiple methods: Interrelationships among measures. Emotion, 5(3), 329-342.

Lynn, R. y Martin, T. (1997). Gender differences in extraversion, neuroticism, and psychoticism in 37 nations. Journal of Social Psychology, 137, 369-373.

MacCann, C. (2006) 'New approaches to measuring emotional intelligence: Exploring methodological issues with two new assessment tools', unpublished doctoral dissertation, University of Sydney, Australia.

Markey, M.A. y Vander-Wal, J.S. (2007). The role of emotional intelligence and negative affect in bulimic symptomatology. Comprehensive Psychiatry, 48 (5), 458-464.

Martinez -Pons, M (1997). The relation of emotional intelligence with selected areas of personal functioning. Imagination, Cognition, and Personality, 17, 3-13.

Martínez, A. E. (2009). Autoconcepto, motivación académica y estrategias de aprendizaje en estudiantes prosociales y no prosociales de educación secundaria obligatoria. Tesis Doctoral. Universidad Miguel Hernández. Elche. España.

Martínez, A.E., Inglés, J.C., Piqueras, J.A. y Ramos V. (2010). Importancia de los amigos y los padres en la salud y el rendimiento académico. Revista Electrónica de Investigación Psicoeducativa / Electronic Journal of Research in Educational Psychology, 20, $8(1), 45-64$.

Martinez-Pons, M. (2000). Emotional intelligence as a self-regulatory process: A social cognitive view. Imagination, Cognition and Personality, 19, 331-350. 
Matthew, G. y Zeidner, M. (2000).Emotional intelligence, adaptation to stressful and health outcomes. En Bar-on, R. y Parker, J.D.A (Eds). The Handbook of Intelligence, JosseyBass.

Matthews, G., Zeidner, Z. y Roberts, R.D. (2002). Emotional intelligence: Science the MIT Press.

Mayer, J. D., Caruso, D. R. y Salovey, P. (1999). Emotional intelligence meets traditional standards for an intelligence. Intelligence, 27, 267-298.

Mayer, J. D., Salovey, P. y Caruso, D. (2000). Models of emotional intelligence (Chap 18). En, R. J. Sternberg (Ed,). Handbook of Intelligence, Cambridge University Press.

Mayer, J. D., Salovey, P. y Caruso, D. (2004). Emotional intelligence: Theory, findings, and implications. Psychological Inquiry, 15, 197-215.

Mayer, J. D., Salovey, P., Caruso, D., y Sitarenios, G. (2003). Measuring emotional intelligence with the MSCEIT V2.0. Emotion, 3, 97-105.

Mayer, J.D., Salovey, P. y Caruso, D. (2002a). Mayer-Salovey-Caruso Emotional Intelligence Test (MS-CEIT), Version 2.0.Toronto:Multi-Health Systems.

McCarthy, C.J., Moller, N.P. y Fouladi, R.T. (2001). Continued attachment to parents: its relationship to affect regulation and perceived stress among college students. Measurement and Evaluation in Counseling and Development, 33, 198-213.

Mikolajczak, M., Petrides, K.V., Coumans, N. y Luminet, O. (2009). The moderating effect of trait emotional intelligence on mood deterioration following laboratory-induced stress. International Journal of Clinical and Health Psychology, 9 (3), 455-477.

Montes-Berges, B. y Augusto, J.-M. (2007). Exploring the relationship between perceived emotional intelligence, coping, social support and mental health in nursing students. Journal of Psychiatric and Mental Health Nursing, 14 (2), 163-171.

Moriarty, N., Stough, C., Tidmarsh, P., Eger, D. y Dennison, S. (2001). Deficits in emotional intelligence underlying adolescent sex offending. Journal of Adolescence, 24, 1-9.

Neisser, U., Boodoo, G., Bouchard, T. J., Boykin, A. W., Brody, N., Ceci, S. J., Halpern, D. F., Loehlin, J. C., Perloff, R., Sternberg, R. J. et Urbina, S. (1996). Intelligence: Knowns and unknowns. American Psychologist, 51, 77-101.

Nelis, D., Quoidbach, J., Mikolajczak, M. y Hansenne M. (2009). Increasing emotional intelligence: (How) is it possible?. Personality and Individual Differences, 47 (1) ,36-41.

Martínez, A.E., Inglés, C., Piqueras, J.A. y Oblitas, L.A. (en prensa). Papel de la conducta prosocial y de las relaciones sociales en el bienestar psíquico y físico del adolescente. Revista Avances en Psicología Latinoamerica. 28,1. 
Neubauer, A.C. y Freudenthaler, H.H. (2005). Models of emotional intelligence. En Schultz, R. $y$ Roberts, R.D. (eds), Emotional intelligence: An international handbook (pp. 3150). Cambridge, MA: Hogrefe.

Nikolaou, I. y Tsaousis, I. (2002). Emotional intelligence in the workplace: Exploring its effects on occupational stress and organizational commitment. Journal of Organizational Analysis, 4, 327-342.

Nolen-Hoeksema, S. (2000).The role of rumination in depressive disorders anxiety/depressive symptoms, Journal of Abnormal psychology, 109, 504-511.

Nolen-Hoeksema, S. (2003).Women who think too much: How to break free of overt reclaim your life. New York: Holt.

Nolen-Hoeksema, S. y Morrow, J. (1991). A prospective study of depression a following a natural disaster: The 1989 Loma Prieta earthquake. Journal of Personality Psychology, 61, 105-121.

Nolen-Hoeksema, S., Larson, J. y Grayson, C. (1999) Explaining the gender difference in depressive symptoms. Journal of Personality and Social Psychology, 77, 1061-1072.

Ogińska-Bulik, N. (2005). Emotional intelligence in the workplace: Exploring its effects on occupational stress and health outcomes in human service workers. International Journal of Occupational Medicine and Environmental Health, 18, 167-175.

Parker, J., Creque, R., Barnhart, D.L., Harris, J.I., Majeski, S.A., Word, L.M.,Bond, B.J., y Hogan, M.J. (2004). Academic achievement in high school: Does emotional intelligence matter? Personality and Individual Differences, 37(7), 1321-1330.

Parkes, K.R. (1990). Coping, negative affectivity and the work environment: Addictive and interactive predictors of mental health, Journal of Applied Psychology, 74, 399-409.

Pau, A. K. H., y Croucher, R. (2003). Emotional intelligence and perceived stress in dental undergraduates. Journal of Dental Education, 67, 1023-1028.

Pecjak, S. (2003). Connection between emotional intelligence and some aspects of psychosocial functioning in elementary and secondary school pupils. Horizons of Psychology, $12(1), 121-139$.

Petrides, K.V., Frederickson, N., y Furnham, A. (2004). The role of trait emotional intelligence in academia performance and deviant behavior at school. Personality and Individual Differences, 36(2), 277-293.

Petrides, K.V., Furnham, A., y Frederickson, N. (2004). Emocional intelligence. Psychologist, $17(10), 574-577$. 
Petrides, K.V., y Furnham, A. (2001). Trait emotional intelligence: Psychometric investigation with reference to established trait taxonomies. European Journal of Personality, $15,425-448$.

Pettit, M.L., Jacob, S.C., Page, K.S. y Porras, C.V. (2010). An assessment of perceived emotional intelligence and eating attitudes among college students. American Journal of Health Education, 41(1),46-52.

Piqueras, J.A., Martínez, A.E., Ramos, V., Rivero, R., García-López, L.J. y Oblitas, L.A. (2008). Ansiedad y depresión: implicaciones para la salud. Suma Psicológica, 15, 4374.

Piqueras, J.A., Ramos, V., Martínez, A.E. y Oblitas, L.A. (2009). Emociones negativas y su impacto en la salud mental y física. Suma psicológica, 16, 85-112.

Queirós, M.M., Cancela, J.M. y Fernández-Berrocal, P. (2004). Inteligencia emocional percibida (IEP) e actividade fisica na terceira idade. Psicologia Educacao Cultura, 8, 187 209.

Ramos, V., Piqueras, J.A., Martínez, A.E. y Oblitas, L.A. (2009). Emoción y Cognición: Implicaciones para el tratamiento. Terapia Psicológica, 2, 1-15.

Ramos, V., Rivero, R., Piqueras, J.A., García-López, L.J. y Oblitas, L.A. (2008). Psiconeuroinmunologia: Conexiones entre Sistema Nervioso y Sistema Inmune. Suma Psicológica, 15, 115-142.

Rector, N. A. y Roger, D. (1997). The stress buffering effects of self-esteem. Personality and Individual Differences, 23, 799-808.

Riley, H., y Schutte, N. (2003). Low emotional intelligence as a predictor of substance-use problems. Journal of Drug Education, 33, 391-398.

Rivero, R., Piqueras, J.A., Ramos, V., García-López, L.J., Martínez, A.E. y Oblitas, L.A. (2008).Psicología y Cancer. Suma Psicológica, 15, 171-198.

Rotter, J. B. (1966). Generalised expectancies for internal versus external control of reinforcement. Psychological Monographs, 80 (1),1-28.

Rude, S.S. y McCarthy, C.T. (2003). Emotional functioning in depressed and depressionvulnerable collage students. Cognition and Emotion, 17, 799-806.

Saklofske, D. H., Austin, E. J., y Minski, P. S. (2003). Factor structure and validity of a trait emotional intelligence measure. Personality and Individual Differences, 34, 707, 721.

Saklofske, D.H., Austin, E.J., Galloway, J. y Davidson K. (2007). Individual difference correlates of health-related behaviours: Preliminary evidence for links between emotional intelligence and coping. Personality and Individual Differences, 42 (3), 491-502. 
Salovey, P. (2001). Applied emotional intelligence: Regulating emotions to become healthy, wealthy, and wise. Emotional intelligence and intimate relationships. En J. Ciarrochi JP. Forgas y JD. Mayer (Eds.) Emotional Intelligence and Everyday Life. New York: Psychology Press.

Salovey, P., Bedell, B., Detweiler, J.B. y Mayer, J. (1999). Coping intelligently intelligence and the coping process. En C.R.Snyder (Eds), Coping: The psychology works (pp.141164).New York: Oxford university press.

Salovey, P., Bedell, B., Detweiler, J.B. y Mayer, J. D. (2000).Current directions in emotional intelligence research. En M. Lewis y J. M. Haviland-Jones (Eds.), Handbook of Emotions (2nd ed.). New York: Guilford Press. Pp. 504-520.

Salovey, P., Mayer, J. D., Goldman, S. L., Turvey, Extremera, N. y Fernández-Berrocal, P. (2002). Relation of perceived emotional intelligence and health-related quality of life of middle-aged women. Psychological Reports, 91, 47-59.

Salovey, P., Mayer, J.D., Goldman, S., Turvey, C. y Palfai, T. (1995). Emotional attention, clarity, and repair: Exploring emotional intelligence using the Trait Mood Scale. En J.W. Pennebaker (Ed.), Emotion, disclosure and health (pp.125-154).Washington DC: American Psychological Association.

Salovey, P., Stroud, L. R., Woolery, A., y Epel, E. S. (2002). Perceived emotional intelligence, stress reactivity, and symptom reports: Further explorations using the trait meta-mood scale. Psychology and Health, 17, 611-627.

Salovey, P., Stroud, L.R., Woolery, A. y Epel, E.S. (2002). Perceived emotional intelligence, stress reactivity, and symptom reports: Further explorations using the Trait MetaMood Scale. Psychology and Health, 17, 611-627.

Salovey, P., y Mayer, J.D. (1990). Emotional intelligence. Imagination Cognition Personality, 9, $185-211$.

Schmidt, J.E. y Andrykowski, M.A. (2004).The role of social and dispositional variables associated with emotional processing in adjustment to breast cancer: an internet-based study. Health Psychology, 23, 259-266.

Schutte N.S., Malouff J.M., Thorsteinsson E.B., Bhullar N. y Rooke S.E. (2007). A metaanalytic investigation of the relationship between emotional intelligence and health.Personality and Individual Differences, 42 (6), 921-933.

Schutte, N.S., Malouff, J.M., Hall, L.E., Haggerty, D.J., Cooper, J.T., Golden, C.J., y Dornheim, L. (1998). Development and validation of a measure of emotional intelligence. Personality and Individual Differences, 25, 167-177. 
Slaski, M. y Cartwright, S. (2000b). Emotional Intelligence Training and its Implications for Stress, Health and Performance Based on paper presented at $3^{\text {rd }}$ World Congress on Stress, September 24-27, Dublin, Ireland.

Slaski, M. y Cartwright, S. (2000a). Health, Performance and Emotional Intelligence: an exploratory study of Retail Managers. Based on paper presented at $3^{\text {rd }}$ World Congress on Stress, September 24-27, Dublin, Ireland.

Slaski, M., y Cartwright, S. (2002). Health, performance and emotional intelligence: An exploratory study of retail managers. Stress and Health, 18, 63-68.

Sukhodolsky, D.G., Golub, A., y Cromwell, E.N. (2001).development and validation of the anger rumination scale. Personality and individual differences, 31, 5, 689-700.

Thayer, J.F., Rossy, L.A., Ruiz-Padial, E. y Johnsen, B.H. (2003). Gender differences in the relationship between Emotional Regulation and Depressive Symptoms. Cognitive Therapy and Research, 27, 349-364.

Trinidad, D.R. y Johnson, C.A. (2002). The association beetween emotional intelligence and early adolescent tobacco and alcohol use. Personality and Individual Differences, 32, 95-105.

Trinidad, D.R., Unger, J.B., Chou, C.P., Azen, S.P. y Johnson, C.A. (2004a). The prospective association of emotional intelligence with psychosocial smoking risk factors for adolescents. Personality and Individual Differences, 36, 945-954.

Trinidad, D.R., Unger, J.B., Chou, C.P., Azen, S.P. y Johnson, C.A. (2004b). Emotional intelligence and smoking risk factors in adolescents: Interactions on smoking intentions. Journal of Adolescent Health, 34, 46-55.

Tsaousis, I., y Nikolaou, I. (2005). Exploring the relationship of emotional intelligence with physical and psychological health functioning. Stress and Health, 21, 77-86.

Van-Rooy, D.L., Alonso, A. y Viswesvaran, Ch. (2005). Group differences in emotional intelligence scores: Theoretical and practical implications. Personality and Individual Differences, 38(3), 689-700.

Velasco, C., Fernández, I., Páez, D. y Campos, M. (2006). Perceived Emotional Intelligence, Alexithymia, Coping and Emotional Regulation. Psicothema, 18, supl., 89-94.

Willard, S. (2006). Relationship of emotional intelligence and adherente to combination antiretroviral medications by individuals living with HIV disease. Journal of the Association of Nurses in Aids Care, 17, 16-26.

Williams, F., Fernández-Berrocal, P., Extremera, N., Ramos, N. y Joiner, T.E. (2004). Mood regulation skill and the symptoms of endogenous and hopelessness depression in 
Spanish high school student. Journal of Psychopathology and Behavioral Assessment, $26,233-240$.

Woodruffe, C. (2001). Promotional Intelligence. People Management, 11 January.

Yalcin, B.M., Karahan, T.F., Ozcelik, M. y Igde F.A. (2008). The effects of an emotional intelligence program on the quality of life and well-being of patients with type 2 diabetes mellitus. Diabetes Educator, 34 (6), 1013-1024.

Yan, J., Wang, L.-J., Cheng, Q., Miao, D.-M., Zhang, L.-Y., Yuan, S.-P., An, T. y Pan, Y. (2008). Estimated mental health and analysis of relative factors for new Chinese recruits. Military Medicine, 173 (10),1031-1034.

Zautra, A.J., Smith, B.W., Affleck, G. y Tennen, H. (2001). Examination of chronic pain and affect relationships from two contrasting approaches: Stress and doping and a dynamic model of affect. Journal of Consulting and Clinical Psychology, 69, 786-795. 